TRANSACTIONS OF THE

AMERICAN MATHEMATICAL SOCIETY

Volume 364, Number 5, May 2012, Pages 2429-2442

S 0002-9947(2012)05470-5

Article electronically published on January 17, 2012

\title{
ON THE ALGEBRAIC FUNDAMENTAL GROUP OF SMOOTH VARIETIES IN CHARACTERISTIC $p>0$
}

\author{
HÉLÈNE ESNAULT AND AMIT HOGADI
}

\begin{abstract}
We define an analog in characteristic $p>0$ of the proalgebraic
\end{abstract} completion of the topological fundamental group of a complex manifold.

\section{INTRODUCTION}

Let $X$ be a smooth algebraic variety defined over a field $k$ endowed with a rational point $x \in X(k)$.

If $k$ is the field of complex numbers $\mathbb{C}$, the proalgebraic completion $\pi^{\text {alg,rs }}(X, x)$ of the topological fundamental group $\pi_{1}^{\text {top }}(X, x)$ is defined as the prosystem $\lim H$, where $H \subset G L(n, \mathbb{C})$ runs over the Zariski closures of the monodromy groups $\rho\left(\pi^{\text {top }}(X, x)\right)$ of complex linear representations $\rho: \pi_{1}^{\text {top }}(X, x) \rightarrow G L(n, \mathbb{C})$. The profinite completion $\lim H$, where $H$ runs over the finite quotients of $\pi_{1}^{\text {top }}(X, x)$, is, via the Riemann existence theorem, identified with Grothendieck's étale fundamental group $\pi_{1}^{\text {ét }}(X, x)$. Since any finite group is embeddable in $G L(n, \mathbb{C})$ for some $n$, this defines, thinking of $\pi_{1}^{\text {et }}(X, x)$ as a complex (constant) proalgebraic group, a surjective homomorphism $\varphi_{\mathbb{C}}^{\mathrm{rs}}: \pi^{\text {alg,rs }}(X, x) \rightarrow \pi_{1}^{\text {ét }}(X, x)$, and in fact $\pi_{1}^{\text {ét }}(X, x)$ is the profinite quotient of $\pi^{\text {alg,rs }}(X, x)$. By the Riemann-Hilbert correspondence, $\pi^{\text {alg,rs }}(X, x)$ is the Tannaka group-scheme of the category of $\mathcal{O}_{X}$-coherent regular singular $\mathcal{D}_{X}$-modules, which is a full subcategory of the category of $\mathcal{O}_{X}$-coherent $\mathcal{D}_{X}$-modules. We denote by $\pi^{\text {alg }}(X, x)$ the corresponding Tannaka group-scheme and by $\varphi_{\mathbb{C}}: \pi^{\text {alg }}(X, x) \rightarrow \pi^{\text {alg,rs }}(X, x) \stackrel{\varphi_{\mathbb{C}}^{\mathrm{rs}}}{\longrightarrow} \pi_{1}^{\text {ét }}(X, x)$ the composite morphism. It is surjective as well, and since any flat connection with finite monodromy is regular singular, $\pi_{1}^{\text {ét }}(X, x)$ is the profinite quotient of $\pi^{\text {alg }}(X, x)$.

If $k$ is a characteristic 0 field, $\pi^{\text {alg }}(X, x)$ is defined as the Tannaka group-scheme of the $k$-linear tensor category of $\mathcal{O}_{X}$-coherent $\mathcal{D}_{X}$-modules equipped with the fiber functor defined as the restriction of the module on $x$. The full subcategory of finite objects, that is, objects with finite monodromy group-scheme or, said differently, objects which have the property that the full Tannaka subcategory which is spanned by it has a finite Tannaka group-scheme and defines a profinite $k$-group-scheme $\pi^{\text {ét }}(X, x)$. Since $\pi^{\text {ét }}(X, x)(\bar{k})=\pi_{1}^{\text {ét }}(X, x)$ ([5, Remark 2.10]) and both $\pi^{\text {alg }}(X, x)$ and $\pi^{\text {ét }}(X, x)$ satisfy base change for finite extensions $k \subset L$ ([6. Property 2.54)]), we see that the surjection $\varphi: \pi^{\text {alg }}(X, x) \rightarrow \pi^{\text {et }}(X, x)$ is a $k$-form of $\varphi_{\mathbb{C}}$ for any complex embedding $k \subset \mathbb{C}$. Moreover, by definition, $\varphi$ induces the profinite quotient homomorphism.

Received by the editors April 11, 2010.

2010 Mathematics Subject Classification. Primary 14F35.

The author was supported by the DFG Leibniz Preis, the SFB/TR45, and the ERC Advanced Grant 226257. 
If $k$ is a characteristic $p>0$ field, the category of $\mathcal{O}_{X}$-coherent $\mathcal{D}_{X}$-modules is again a $k$-linear abelian tensor rigid category. It is part of Katz' theorem asserting that this category is equivalent to the category of stratified $\mathcal{O}_{X}$-coherent sheaves (see [9, Theorem 1.3], [3, Theorem 8], where it is shown over $k=\bar{k}$ ). If $k=\bar{k}$, its Tannaka group-scheme $\pi^{\text {alg }}(X, x)$ is shown to be prosmooth in [3, Corollary 12] (strictly speaking, it is shown there only for the profinite part, but dos Santos' proof applies more generally as mentioned in [4, Corollary 7]). The homomorphism $\varphi$ is then defined by the full embedding of the subcategory of objects with finite monodromy group-scheme. So by definition, $\varphi$ induces the profinite quotient homomorphism.

On the other hand, if $X$ is a reduced connected scheme over a characteristic $p>0$ field $k$, endowed with a rational point $x \in X(k)$, Nori [10, Chapter II] constructed a fundamental group-scheme $\pi^{N}(X, x)$ as the projective system of finite $k$-groupschemes $G$ for which there is a $G$-torsor $h: Y \rightarrow X$ under $G$ with trivialization at $x$. The proétale quotient of $\pi^{N}(X, x)$ is precisely $\pi^{\text {ét }}(X, x)$.

Summarizing, one has a diagram

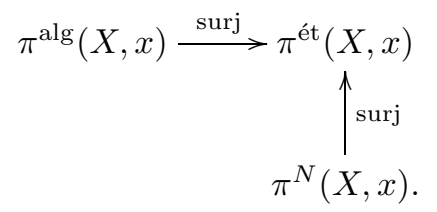

The aim of our article is to define a Tannaka category $\operatorname{Strat}(X, \infty)$ over a perfect field $k$, which contains the category of $\mathcal{O}_{X}$-coherent $\mathcal{D}_{X}$-modules as a full subcategory, in such a way that its Tannaka group-scheme $\pi^{\text {alg, } \infty}(X, x)$, which thus surjects onto $\pi^{\text {alg }}(X, x)$, also surjects onto $\pi^{N}(X, x)$. In other words, we complete (1.1) to

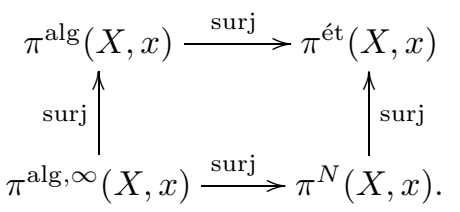

As a byproduct, we obtain a purely Tannakian geometric description of $\pi^{N}(X, x)$ (see Corollary 4.9). Recall that we assume that $X$ is smooth. If in addition $X$ is proper, Nori himself described his fundamental group-scheme $\pi^{N}(X, x)$ as the Tannaka group-scheme of the category of essentially finite bundles [10, Chapter I]. He extends in [10, Chapter III] his construction to non-proper curves by using parabolic bundles. Lacking desingularization in characteristic $p>0$ makes it difficult to generalize his construction to the higher dimensional case. If $k$ has characteristic 0 , then, as already mentioned, $\pi^{N}(X, x)=\pi^{\text {ét }}(X, x)$ is the Tannaka group-scheme of the category of finite flat connections [6, Section 2] or, equivalently, of the category of $\mathcal{O}_{X}$-coherent $\mathcal{D}_{X}$-modules with finite monodromy group-scheme.

Our construction (see Section 3, most particularly Definition 3.2) generalizes on a smooth variety defined over a perfect characteristic $p>0$ field $k$ the construction of the category of flat connections (loc. cit) in characteristic 0 and the construction of the stratified bundles (loc. cit.) in characteristic $p>0$. We now explain the main idea.

For $i \in \mathbb{N}$, let us define inductively the relative Frobenius $F^{(i)}: X^{(i)} \rightarrow X^{(i+1)}$ over $k$ in the usual manner. As $k$ is assumed to be perfect, one defines $X^{(-1)}=$ 
$X \otimes_{k, F_{k}^{-1}} k$, where $F_{k}:$ Spec $k \rightarrow$ Spec $k$ is the absolute Frobenius of $k$, together with the relative Frobenius $F^{(-1)}: X^{(-1)} \rightarrow X^{(0)}$. Then one iterates to define inductively $F^{(i)}: X^{(i)} \rightarrow X^{(i+1)}$ for $i \in \mathbb{Z}, i<0$. For $a, b \in \mathbb{Z}, a<b$ we define $F^{(a, b)}: X^{(a)} \stackrel{F^{(a)} \circ \ldots \circ F^{(b-1)}}{\longrightarrow} X^{(b)}$.

Recall that a stratified bundle is a sequence $\left(E^{(i)}, \sigma^{(i)}, i \in \mathbb{N}\right)$, where $E^{(i)}$ is a bundle on $X^{(i)}$ and $\sigma^{(i)}: E^{(i)} \cong F^{(i) *} E^{(i+1)}$ is a $\mathcal{O}_{X^{(i)}}$-isomorphism. For $t \in$ $\mathbb{N}, t \neq 0$, we define an object of $\operatorname{Strat}(X, t)$ to be a sequence $\left(E^{(i)}, \sigma^{(i)}, i \in \mathbb{N}\right)$, where $E^{(i)}$ is a bundle on $X^{(i)}$ and $\sigma^{(i)}: E^{(i)} \stackrel{\cong}{\longrightarrow} F^{(i) *} E^{(i+1)}$ is a $\mathcal{O}_{X^{(i)}}$-isomorphism for all $i \geq 1$, but for $i=0, \sigma_{0}: F^{(-t, 0) *} E^{(0)} \cong F^{(-t, 1) *} E^{(1)}$ is a $\mathcal{O}_{X^{(-t)}}$-isomorphism. The morphisms are the ones between the bundles which respect all the structures. We show (Theorem 3.4) that the obvious functor Strat $(X, t) \subset \operatorname{Strat}(X, t+1)$, which assigns $\left(E_{i}, F^{(-t-1) *} \sigma_{0}, \sigma_{i}, i \geq 1\right)$ to $\left(E_{i}, \sigma_{0}, \sigma_{i}, i \geq 1\right)$, induces a full embedding of Tannaka categories, where the fiber functor is simply the restriction of $E^{(0)}$ to the rational point $x$. Then $\operatorname{Strat}(X, \infty)$ is defined as the inductive limit over $t \rightarrow \infty$ of the categories $\operatorname{Strat}(X, t)$ (Corollary 3.5). In order to show that the Tannaka group-scheme $\pi^{\text {alg, } \infty}(X, x)$ of $\operatorname{Strat}(X, \infty)$ surjects onto $\pi^{N}(X, x)$, we use a slight modification of Nori's reconstruction theorem [10, Chapter I, Proposition 2.9] of a torsor $h: Y \rightarrow X$ under a finite group scheme $G$ out of the induced functor $h^{\#}$ : $\operatorname{Rep}_{k}(G) \rightarrow \operatorname{Coh}(X)$ which assigns to a finite dimensional $k$-linear representation $V$ of $G$ the vector bundle on $X$ which is defined by flat descent for $h$ on $\mathcal{O}_{Y} \times_{k} V$ (Theorem 2.4).

This allows us to define the group-scheme homomorphism $\pi^{\text {alg, } \infty}(X, x) \rightarrow$ $\pi^{N}(X, x)$ (Theorem 4.5). In order to show that this map induces the profinite quotient, we in particular use the categorial translation of injectivity and surjectivity of homomorphisms of Tannaka group-schemes ([2, Proposition 2.12]).

\section{Nori's Fundamental GROUP-SCHEME}

Let $k$ be a field of characteristic $p>0$ and $X$ be a $k$-scheme. Let $x \in X(k)$ be a rational point and $i_{x}: x \rightarrow X$ be the closed embedding.

Nori [10, Chapter II] defines the category $\mathrm{N}(X, x)$ of triples $(Y \stackrel{f}{\rightarrow} X, G, y)$ where

(a) $G / k$ is a finite group scheme,

(b) $f: Y \rightarrow X$ is a $G$-torsor,

(c) $y$ is a $k$-point of $Y$ lying above $x$.

A morphism between two such triples $\left(Y_{i} \stackrel{f_{i}}{\rightarrow} X, G_{i}, y_{i}\right), i=1,2$, is a pair $(\phi$ : $\left.G_{1} \rightarrow G_{2}, \psi: Y_{1} \rightarrow Y_{2}\right)$ such that $\psi$ is an $X$-morphism which is $\phi$-equivariant and $\psi\left(y_{1}\right)=y_{2}$. Nori shows [10, Chapter II, Proposition 2] that if $X$ is reduced and geometrically connected, then the projective limit $\lim _{\leftarrow} G$ exists. He defines

$$
\mathrm{N}(X, x)
$$

Definition 2.1. Let $X$ be a reduced geometrically connected $k$-scheme. Then its Nori fundamental group-scheme is the profinite $k$-group-scheme

$$
\pi^{N}(X, x)=\lim _{\mathrm{N}(X, x)} G
$$

Since giving a rational point $y \in f^{-1}(x)$ is the same as giving a trivialization $f^{-1}(x) \cong_{k} G, \mathrm{~N}(X, x)$ is equivalent to the category of triples ( $h: Y \rightarrow$ $\left.X, G, f^{-1}(x) \cong_{k} G\right)$, where the morphisms between two such objects are defined by 
torsor morphisms which respect the trivialization. We will not need this slightly different phrasing.

Definition 2.2. Let $G$ be a finite $k$-group-scheme, and let $h: Y \rightarrow X$ be a $G$ torsor. Then it induces a functor $h^{\#}: \operatorname{Rep}_{k}(G) \rightarrow \operatorname{Coh}(X)$ which assigns to a finite dimensional $k$-representation $V$ the bundle on $X$ which comes by flat descent from $\mathcal{O}_{Y} \otimes_{k} V$.

Properties 2.3. $\quad$ 1) The functor $h^{\#}$ defined in Definition 2.2 is exact, $k$-linear and compatible with the tensor structure. Thus it is a fiber functor in the sense of Deligne [1, 1.9]. Since $\operatorname{Rep}_{k}(G)$ is a Tannaka category, it follows [1, Corollaire 2.10] that $h^{\#}$ is faithful.

2) The functor $i_{x}^{*}: \operatorname{Coh}(X) \rightarrow \operatorname{Vec}_{k}$ defined as the restriction to the rational point, with values in the category of finite dimensional $k$-vector spaces, is a fiber functor on the subcategory of vector bundles. The composite functor $i_{x}^{*} \circ h^{\#}: \operatorname{Rep}_{k}(G) \rightarrow \operatorname{Vec}_{k}$ is a fiber functor.

3) Let $h_{i}: Y_{i} \rightarrow X$ be $G_{i}$-torsors where $i=1,2$. Let $\phi: G_{1} \rightarrow G_{2}$ be a group homomorphism and $\psi: Y_{1} \rightarrow Y_{2}$ be an equivariant map with respect to $\phi$. We denote by $\phi^{*}$ the induced functor $\operatorname{Rep}_{k}\left(G_{2}\right) \rightarrow \operatorname{Rep}_{k}\left(G_{1}\right)$. Then one has the equality $h_{2}^{\#}=h_{1}^{\#} \circ \phi^{*}$ of functors. Indeed, if $V$ is a $G_{2^{-}}$ representation, $\psi^{*}: \mathcal{O}_{Y_{2}} \otimes_{k} V \rightarrow \psi_{*}\left(\mathcal{O}_{Y_{1}} \otimes_{k} \phi^{*}(V)\right)$ induces a $\mathcal{O}_{X}$-linear map $h_{2}^{\#}(V) \rightarrow h_{1}^{\#}(V)$ between those two vector bundles, which, after composing with $i_{x}^{*}$, is the identity on $V$. So $h_{2}^{\#}(V)=h_{1}^{\#} \circ \phi^{*}(V)$.

4) Let $h: Y \rightarrow X$ be a $G$-torsor, let $b: X^{\prime} \rightarrow X$ be a morphism, and let $x^{\prime} \in X^{\prime}(k)$ be a rational point with $b\left(x^{\prime}\right)=x$. Let $Y^{\prime}=Y \times_{X} X^{\prime} \rightarrow X^{\prime}$ and $h^{\prime}: Y^{\prime} \rightarrow X^{\prime}$ denote the projection. Then one has the equality $b^{*} \circ h^{\#}=h^{\prime \#}$ of functors. Indeed, denoting by $b^{\prime}: Y^{\prime} \rightarrow Y$ the induced morphism, if $V$ is a $G$-representation, $\left(b^{\prime}\right)^{*}: \mathcal{O}_{Y} \otimes_{k} V \rightarrow\left(b^{\prime}\right)_{*} \mathcal{O}_{Y^{\prime}} \otimes_{k} V$ induces $\mathcal{O}_{X^{\prime}}$-linear map $b^{*} \circ h^{\#}(V) \rightarrow\left(h^{\prime}\right)^{\#}(V)$ between vector bundles, which is the identity on $V$ after composing with $i_{x^{\prime}}$. So $b^{*} \circ h^{\#}=\left(h^{\prime}\right)^{\#}$.

The following is a direct consequence of [10, Proposition 2.9].

Theorem 2.4. Let $G$ be a finite k-group-scheme and let $F: \operatorname{Rep}_{k}(G) \rightarrow \operatorname{Coh}(X)$ be a fiber functor such that $i_{x}^{*} \circ F$ is the forgetful functor $F_{G}: \operatorname{Rep}_{k}(G) \rightarrow \operatorname{Vec}_{k}$. Then there exists a unique object $(Y \stackrel{h}{\rightarrow} X, G, y)$ of $\mathrm{N}(X, x)$ such that $F=h^{\#}$ and $\left(h^{-1}(x), y\right)=(G, 1)$. For any other object $\left(Y^{\prime} \stackrel{h^{\prime}}{\rightarrow} X, G, y^{\prime}\right) \in \mathrm{N}(X, x)$ such that $F=h^{\#}$, there exists a unique isomorphism in $\mathrm{N}(X, x)$ between $(Y \stackrel{h}{\rightarrow} X, G, y)$ and $\left(Y^{\prime} \stackrel{h^{\prime}}{\rightarrow} X, G, y^{\prime}\right)$.

Proof. By Nori's reconstruction theorem [10, Proposition 2.9], $F(k[G])$, where $k[G]$ is the regular representaton of $G$, is a finite $\mathcal{O}_{X}$-algebra. The $G$-torsor $h: Y \rightarrow X$ is defined to be $\operatorname{Spec}_{X} F(k[G])$. By Property $[2.32), i_{x}^{*} \circ F(k[G])=F_{G}(k[G])=$ $k[G]$. Said differently, $h^{-1}(x)=\operatorname{Spec}_{x} k[G]=G$. Then $y$ is the rational point of $h^{-1}(x)$ which is $1 \in G$. By the unicity in loc. cit., $h$ is uniquely defined. If $y^{\prime}=g \in h^{-1}(x)(k)$ is another rational point, then multiplication $g: Y \rightarrow Y$ by $g$, together with the conjugation $G \rightarrow G, h \mapsto g h g^{-1}$, defines an isomorphism $(h: Y \rightarrow X, G, y) \rightarrow\left(h: Y \rightarrow X, G, y^{\prime}\right)$ in $\mathrm{N}(X, x)$. 


\section{THE CATEGORY OF GENERALIZED STRATIFIED BUNDLES}

The aim of this section is to define the category of generalized stratified bundles. We start with some notation.

Notation 3.1. Let $k$ be a perfect field of characteristic $p>0$ and let $X$ be a smooth scheme over $k$ which is geometrically irreducible.

For $i \in \mathbb{N}$, we define inductively the relative Frobenius $F^{(i)}: X^{(i)} \rightarrow X^{(i+1)}$ over $k$ in the usual manner, by defining $X^{(0)}=X, X^{(i+1)}$ to be the fiber product of $X^{(i)} \otimes_{k, F_{k}} k$ over the absolute Frobenius $F_{k}:$ Spec $k \rightarrow \operatorname{Spec} k$ of $k$ and defining $F^{(i)}$ to be the factorization of the absolute Frobenius $F_{X^{(i)}}: X^{(i)} \rightarrow X^{(i)}$ morphism.

For $i \in \mathbb{Z}, i<0$, we define inductively $F^{(i)}: X^{(i)} \rightarrow X^{(i+1)}$ over $k$ as follows. First we set $X^{(-1)}=X \otimes_{F_{k}^{-1}} k$. Then we define $F^{(-1)}: X^{(-1)} \rightarrow X$ to be the relative Frobenius. Similarly, we define $X^{(-i-1)}=X^{(-i)} \otimes_{F_{k}^{-1}} k$, together with the relative Frobenius $F^{(-i-1)}: X^{(-i-1)} \rightarrow X^{(-i)}$ over $k$.

For $a, b \in \mathbb{Z}, a<b$ we define $F^{(a, b)}: X^{(a)} \stackrel{F^{(a)} \circ \ldots \circ F^{(b-1)}}{\longrightarrow} X^{(b)}$.

Recall that a stratified bundle (see [9, Section 1]) is a sequence $\left(E^{(i)}, \sigma^{(i)}\right), i \in \mathbb{N}$, where $E^{(i)}$ is a $\mathcal{O}_{X^{-}}$coherent sheaf on $X^{(i)}$ and $\sigma^{(i)}: E^{(i)} \cong F^{(i) *} E^{(i+1)}$ is a $\mathcal{O}_{X^{(i)}}$ isomorphism. One defines the category $\operatorname{Strat}(X)$ of stratified bundles by defining

$$
\operatorname{Hom}\left(\left(D^{(i)}, \tau^{(i)}\right),\left(E^{(i)}, \sigma^{(i)}\right)\right)
$$

to be a set of sequences $f_{i}: D^{(i)} \rightarrow E^{(i)}$ of morphisms of $\mathcal{O}_{X^{(i)}}$-coherent sheaves, which commute with all the $\sigma_{i}$ and $\tau_{i}$. It is a fact (loc. cit.) that if $\left(E^{(i)}, \sigma^{(i)}, i \in \mathbb{N}\right)$ is a stratified sheaf, the $E^{(i)}$ are all locally free, and if $f=(f)_{i}, i \in \mathbb{N}$, is a morphism of stratified sheaves, then $f_{i}$ are vector bundle maps (i.e. locally split), so the categroy is abelian, rigid, and monoidal. Moreover, the Hom-sets are finite dimensional $k$-vector spaces. As $X$ is geometrically irreducible, the unit object $\mathbb{I}=$ $\left(\mathcal{O}_{X}\right.$, Id $), i \in \mathbb{N}$, fulfills $\operatorname{End}(\mathbb{I})=k$. If now $X$ is endowed with a rational point $x \in$ $X(k)$, then $\omega_{x}$ : Strat $(X) \rightarrow \operatorname{Vec}_{k},\left.\left(E^{(i)}, \sigma^{(i)}\right) \mapsto E_{0}\right|_{x}$ is a fiber functor in the sense of Deligne [1, 1.9] and thus yields the structure of a Tannaka category on $\operatorname{Strat}(X)$. A fundamental property due to dos Santos is that the corresponding Tannaka $k$ group-scheme $\operatorname{Aut}^{\otimes}\left(\omega_{x}\right)$ is prosmooth ([3, Corollary 12], [4, Corollary 7]).

Definition 3.2. Let $t \geq 0$ be an integer. A t-stratified bundle is a sequence

$$
\left(E^{(i)}, \sigma^{(i)}, i \in \mathbb{N}\right) \text {, }
$$

where $E^{(i)}$ is a $\mathcal{O}_{X}$-coherent sheaf on $X^{(i)}$,

$$
\sigma^{(i)}: E^{(i)} \cong F^{(i) *} E^{(i+1)}
$$

is a $\mathcal{O}_{X^{(i)}}$-isomorphism for $i \geq 1$, and for $i=0$,

$$
\sigma^{(0)}: F^{(-t, 0) *} E^{(0)} \cong F^{(-t, 1) *} E^{(1)}
$$

is a $\mathcal{O}_{X^{(-t)}}$-isomorphism.

One defines the category $\operatorname{Strat}(X, t)$ of $t$-stratified bundles by defining

$$
\operatorname{Hom}\left(\left(D^{(i)}, \sigma^{(i)}\right),\left(E^{(i)}, \tau^{(i)}\right)\right)
$$

to be a set of sequences $f_{i}: D^{(i)} \rightarrow E^{(i)}$ of morphisms of $\mathcal{O}_{X}$-coherent sheaves, which commute with all the $\sigma_{i}$ and $\tau_{i}$.

In particular, $\operatorname{Strat}(X, 0)=\operatorname{Strat}(X)$. 
Example 3.3. We now give an example of a non-trivial 1-stratified bundle on $X=\mathbb{A}_{k}^{1}=\operatorname{Spec}\left(k([x])\right.$. Thus $X^{(i)}=\operatorname{Spec}\left(k\left[x_{i}\right]\right)$, where the relative Frobenius $X^{(i)} \rightarrow X^{(i+1)}$ is induced by $x_{i+1} \rightarrow x_{i}^{p}$. For simplicity let us assume $p=\operatorname{char}(k)=$ 2 . Let $V$ be a 2 dimensional vector space over $k$ with basis $e_{1}, e_{2}$. Define

$$
E^{(i)}=\mathcal{O}_{X^{(i)}} \otimes_{k} V \quad \forall i \geq 0
$$

and

$$
\sigma^{(i)}: E^{(i)} \rightarrow F^{(i) *} E^{(i+1)}, \quad i \geq 1,
$$

to be the isomorphism induced by the identity on $V$. We define

$$
\sigma^{(0)}: F^{(-1,0) *} E^{(0)} \rightarrow F^{(-1,1) *} E^{(1)}
$$

to be the isomorphism defined by sending

$$
e_{1} \rightarrow e_{1}, \quad e_{2} \rightarrow x_{-1} e_{1}+e_{2} .
$$

We claim that the -1 -stratified bundle thus defined is not isomorphic to the trivial stratified bundle of rank 2. If indeed this were the case, then we would have a $k[x]$-module automorphism $\phi: k[x] \otimes_{k} V \rightarrow k[x] \otimes_{k} V$ such that

$$
\phi \otimes_{k[x]} k\left[x_{-1}\right]=\sigma^{(0)} .
$$

This is impossible since $x_{-1}$ is not contained in $k[x]$. It can be shown (see (4.3) that this -1-stratified bundle "arises" from the non-trivial $\alpha_{p}$-torsor on $\mathbb{A}_{k}^{1}$ defined by the relative Frobenius of $\mathbb{A}_{k}^{1}$.

Theorem 3.4. The notation is as in Notation 3.1.

1) For every integer $t \geq 0$, Strat $(X, t)$ is a $k$-linear, abelian, rigid, tensor category.

2) The functor

$$
\begin{gathered}
(+): \operatorname{Strat}(X, t) \subset \operatorname{Strat}(X, t+1), \\
\left(E_{i}, \sigma_{0}, \sigma_{i}, i \geq 1\right) \mapsto\left(E_{i}, F^{(-t-1) *} \sigma_{0}, \sigma_{i}, i \geq 1\right)
\end{gathered}
$$

induces a full faithful embedding of k-linear, abelian, rigid, tensor categories.

3) If $x \in X(k)$ is a rational point, the functor

$$
\begin{gathered}
\omega_{x}: \operatorname{Strat}(X, t) \rightarrow \operatorname{Vec}_{k}, \\
\left.\left(E^{(i)}, \sigma^{(i)}\right) \mapsto E_{0}\right|_{x}
\end{gathered}
$$

is a fiber functor, which makes (Strat $\left.(X, t), \omega_{x}\right)$ a Tannaka category.

Proof. We show 1). Since $\operatorname{Strat}(X, 0)=\operatorname{Strat}(X)$, we assume $t>0$. If $\left(E^{(i)}, \sigma^{(i)}, i \in\right.$ $\mathbb{N})$ is an object in $\operatorname{Strat}(X, t)$, then $\left(E_{+}^{(i)}=E^{(i+1)}, \sigma_{+}^{(i)}=\sigma^{(i+1)}, i \in \mathbb{N}\right)$ is an object $\operatorname{Ver}\left(E^{(i)}, \sigma^{(i)}, i \in \mathbb{N}\right) \in \operatorname{Strat}\left(X^{(1)}\right)$. Since $E^{(i)}$ is locally free, by the isomorphism $\sigma^{(0)}, F^{(-t, 0) *} E^{(0)}$ is locally free. Since $X$ is smooth, the relative Frobenius is flat; thus by flat descent, $E^{(0)}$ is locally free as well. So $\operatorname{Strat}(X)$ is rigid and monoidal. On the other hand,

$$
\begin{aligned}
\operatorname{Hom}\left(\left(D^{(i)}, \tau^{(i)}, i \in \mathbb{N}\right)\right. & \left.,\left(E^{(i)}, \sigma^{(i)}, i \in \mathbb{N}\right)\right) \\
& \subset \operatorname{Hom}\left(\operatorname{Ver}\left(D^{(i)}, \tau^{(i)}, i \in \mathbb{N}\right), \operatorname{Ver}\left(E^{(i)}, \sigma^{(i)}, i \in \mathbb{N}\right)\right)
\end{aligned}
$$

and is obviously a $k$-vector space. So the Hom-sets are finite dimensional $k$-vector spaces. Moreover, any morphism $f=\left(f^{(i)}, i \in \mathbb{N}\right)$ is such that $f^{i}, i \geq 1$, is a 
morphism of vector bundles. Thus by the isomorphisms $\tau^{(0)}$ and $\sigma^{0}$, Ker, Im and Coker of $f^{(0)}$ are pulled back to vector bundles on $X^{(-t)}$ via $F^{(-t, 0)}$. Thus by flat descent again, there are vector bundles on $X$. We conclude that $\operatorname{Strat}(X, t)$ is an abelian category. This shows 1 ).

2) follows immediately from the factorization of (3.1) through $(+)$.

We show 3): the point $x \in X(k)$ maps to $x^{(1)} \in X^{(1)}(k)$, and the map $x \rightarrow x^{(1)}$ is the identity on the residue fields $k(x)=k\left(x^{(1)}\right)=k$. If $0 \rightarrow A \rightarrow B \rightarrow C \rightarrow 0$ is an exact sequence in $\operatorname{Strat}(X, t)$, then $0 \rightarrow \operatorname{Ver}(A) \rightarrow \operatorname{Ver}(B) \rightarrow \operatorname{Ver}(C) \rightarrow 0$ is an exact sequence in $\operatorname{Strat}\left(X^{(1)}\right)$. Thus $0 \rightarrow \omega_{x^{(1)}}(\operatorname{Ver}(A)) \rightarrow \omega_{x^{(1)}}(\operatorname{Ver}(B)) \rightarrow$ $\omega_{x^{(1)}}(\operatorname{Ver}(C)) \rightarrow 0$ is an exact sequence in $\operatorname{Vec}_{k}$. But

$$
\omega_{x^{(1)}}(\operatorname{Ver}(A))=\omega_{x}(A) .
$$

This shows that $\omega_{x}$ is exact. Furthermore, $\omega_{x}$ is obviously $k$-linear and compatible with the tensor structure. This finishes the proof.

Corollary 3.5. Let the notation be as in Theorem 3.4. The category

$$
\operatorname{Strat}(X, \infty)=\underset{+, t \in \mathbb{N}}{\lim _{+, t}} \operatorname{Strat}(X, t)
$$

is a $k$-linear, abelian, rigid tensor category on which, if $X$ has a rational point $x \in X(k)$, the functor $\omega_{x}$ is a fiber functor.

Definition 3.6. The notation is as in Theorem 3.4.

1) We define $\pi^{\text {alg }}(X, x)$ to be the Tannaka $k$-group scheme $\operatorname{Aut}^{\otimes}\left(\omega_{x}\right)$ of $\left(\operatorname{Strat}(X), \omega_{x}\right)$.

2) We define $\pi^{\text {alg, } \infty}(X, x)$ to be the Tannaka $k$-group scheme $\operatorname{Aut}^{\otimes}\left(\omega_{x}\right)$ of $\left(\operatorname{Strat}(X, \infty), \omega_{x}\right)$.

The functor $(+): \operatorname{Strat}(X) \rightarrow \operatorname{Strat}(X, \infty)$ defines the homomorphism

$$
(+)^{*}: \pi^{\text {alg }, \infty}(X, x) \rightarrow \pi^{\text {alg }}(X, x) .
$$

Lemma 3.7. The homomorphism $(+)^{*}$ in (3.3) is faithfully flat.

Proof. We apply [2, Proposition 2.21]. As $(+)$ is fully faithful, the lemma is equivalent to saying that if $A$ is an object on $\operatorname{Strat}(X)$ and $B \subset(+) A$ is a subobject in $\operatorname{Strat}(X, \infty)$, then there is a subobject $B^{\prime} \subset A$ in $\operatorname{Strat}(X)$ such that $B=(+) B^{\prime}$. One has that $\operatorname{Ver}(B) \subset \operatorname{Ver}(A)$ is a subobject in $\operatorname{Strat}\left(X^{(1)}\right)$. Thus $F^{(0) *} B^{(1)} \subset A^{(0)}$ is a subvector bundle with the property that $F^{(-t, 0) *} \circ F^{(0) *} B^{(1)}=F^{(-t, 1) *} B^{(1)}=$ $F^{(-t, 0) *} B^{(0)}$. Thus $B^{\prime}=\left(F^{(0) *} B^{(1)}, B^{(i)}, i \geq 1, F^{(0) *}, \sigma^{(i)}, i \geq 1\right) \subset A$ is a subobject of $A$ such that $(+) B^{\prime}=B$. This finishes the proof.

\section{Comparison of $\pi^{\mathrm{alg}, \infty}(X, x)$ With $\pi_{1}^{N}(X, x)$}

In order to achieve the comparison, we start with a construction.

Construction 4.1. The notation is as in Notation 3.1, and $x \in X(k)$ is a rational point. Let $(h: Y \rightarrow X, G, y)$ be an object of $\mathrm{N}(X, x)$. Using this object, we construct a tensor functor

$$
h^{*}: \operatorname{Rep}_{k}(G) \rightarrow \operatorname{Strat}(X, \infty)
$$


together with a factorization of functors

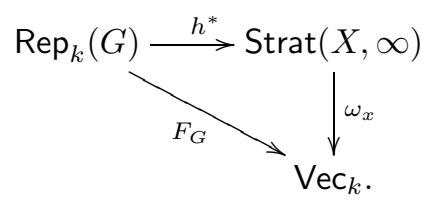

Here $F_{G}: \operatorname{Rep}_{k}(G) \rightarrow \operatorname{Vec}_{k}$ is the forgetful functor.

Recall that if $G$ is a finite $k$-group-scheme, there is an exact sequence of finite $k$-group schemes $1 \rightarrow G_{0} \rightarrow G \rightarrow G_{\text {ét }} \rightarrow 1$, where $G_{0}$ is the 1-component of $G$ and $G_{\text {ét }}$ is étale. Furthermore, as $k$ is perfect, $G_{\text {red }} \subset G$ is a closed subgroup-scheme and the composite $G_{\text {red }} \stackrel{\iota}{\rightarrow} G \rightarrow G_{\text {ét }}$ is an isomorphism. Thus $\iota$ yields on $G$ the structure of a semi-direct product of $G_{\text {ét }}$ by $G_{0}$. The construction of $h^{*}$ will be such that the image of $h^{*}$ is contained in $\operatorname{Strat}(X, t)$, where $t$ is a natural number such that the image of the $k$-group-scheme homomorphism $G^{(-t)} \rightarrow G$ is equal to $G_{\text {ét }}$.

Let $V$ be a finite dimensional $k$-representation of $G$. We set

$$
E^{(0)}=h^{\#}(V) .
$$

For $i \in \mathbb{N} \backslash\{0\}$, the relative Frobenius is an isomorphism of the étale $k$-groupschemes

$$
F^{(0, i)}: G_{\text {ét }} \cong G_{\text {ét }}^{(i)} .
$$

Thus $\iota(G) \circ F^{(0, i)-1}: G_{\text {ét }}^{(i)} \subset G$ is a closed embedding, and composing with it defines a $G_{\text {ét }}^{(i)}$-action on $V$. Since $h: Y \rightarrow X$ is a $G$-torsor, for $i \geq 0, h^{(i)}: Y^{(i)} \rightarrow X^{(i)}$ is also a $G^{(i)}$-torsor. Let $h_{\text {ét }}^{(i)}: Y_{\text {ét }}^{(i)} \rightarrow X^{(i)}$ be the induced $G_{\text {ét }}^{(i)}$-torsor obtained by moding out by $G_{0}^{(i)}$. We define

$$
E^{(i)}=\left(h_{\text {ét }}^{(i)}\right)^{\#}(V) .
$$

One has

$$
\sigma^{(i)}: E^{(i)} \equiv F^{(i) *} E^{(i+1)}, i \in \mathbb{N} \backslash\{0\} .
$$

The object $h^{*}(V) \in \operatorname{Strat}(X, t)$ which we wish to construct will have the property

$$
\operatorname{Ver}\left(h^{*}(V)\right)=\left(E^{(i)}, \sigma^{(i)}, i \geq 1\right) .
$$

It remains to define $\sigma^{(0)}$. By definition,

$$
F^{(0) *} E^{(1)}=\left(h_{\text {ét }}^{(0)}\right)^{\#}(V)=\left(h_{\text {ét }}\right)^{\#}(V) .
$$

Let $t$ be a natural number such that the image of $G^{(-t)} \rightarrow G$ is equal to $G_{\text {ét. }}$. One has the following commutative diagram of $k$-varieties:

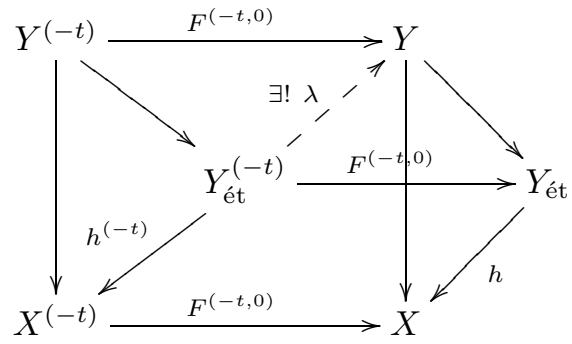


The morphism $F^{(-t, 0)}: Y^{(-t)} \rightarrow Y$ is equivariant under $F^{(-t, 0)}: G^{(-t)} \rightarrow G$. Likewise, the morphism $F^{(-t, 0)}: Y_{\text {ét }}^{(-t)} \rightarrow Y_{\text {ét }}$ is equivariant under $F^{(-t, 0)}: G_{\text {ét }}^{(-t)} \rightarrow$ $G_{\text {ét }}$. The commutativity of the diagram implies that

$$
\lambda^{*}\left(\mathcal{O}_{Y} \otimes_{k} V\right)=F^{(-t, 0) *}\left(\mathcal{O}_{Y_{\text {ét }}} \otimes_{k} V\right)=F^{(-t, 1) *}\left(\mathcal{O}_{Y_{\text {êt }}^{(1)}} \otimes_{k} V\right)
$$

equivariantly for the action of $G_{\text {ét }}^{(-t)}$. Thus

$$
\left(h_{\text {ét }}^{(-t)}\right)^{\#}(V)=F^{(-t, 0) *} E^{(0)}=F^{(-t, 1) *} E^{(1)} .
$$

We define $\sigma^{(0)}: F^{(-t, 0) *} E^{(0)}=F^{(-t, 1) *} E^{(1)}$ to be the equality of (4.10).

Thus, starting with $V \in \operatorname{Rep}_{k}(G)$, we have constructed an object $h^{*}(V)=$ $\left(E^{(i)}, \sigma^{(i)}, i \in \mathbb{N}\right) \in \operatorname{Strat}(X, t)$. Clearly, any $\phi \in \operatorname{Hom}_{\operatorname{Rep}_{k}(G)}(V, W)$ induces $h^{*}(\phi) \in$ $\operatorname{Hom}_{\operatorname{Strat}(X, t)}\left(h^{*}(V), h^{*}(W)\right)$. This defines the functor

$$
h^{*}: \operatorname{Rep}_{k}(G) \rightarrow \operatorname{Strat}(X, \infty)
$$

by composing with $(+)$. Moreover, one has

$$
h^{*}(V)_{x}=\left(\mathcal{O}_{Y} \otimes_{k} V\right)_{y}=V .
$$

This shows the commutativity of (4.1).

Remark 4.2. In the above construction we use the fact that for a finite flat group scheme $G$ over a perfect field $k$, the epimorphism $G \rightarrow G_{\text {ét }}$ admits a section (necessarily unique). In other words $G_{\text {ét }}$ can be canonically thought of as a subgroup scheme of $G$ via the identification $G_{\text {red }}=G_{\text {ét }}$. When $k$ is not a perfect field, $G_{\text {red }}$ may not be a subgroup scheme (for example, $G=\operatorname{Spec} k[t] /\left(t^{p^{2}}-a t^{p}\right), a \in k \backslash k^{p}$; see [8. Chapter III, Exercice (3.2)]) and the above construction of $h^{*}$ does not make sense. This is the reason why we assume throughout that $k$ be perfect. We thank Nguyên Duy Tân for this important remark.

Example 4.3. Let $p=\operatorname{char}(k)=2$ for simplicity and let $G=\alpha_{2}=\operatorname{Spec}\left(k[t] / t^{2}\right)$. Let $X=\mathbb{A}_{k}^{1}=\operatorname{Spec}(k[x])$. Let $P=\operatorname{Spec}(k[u])$ and $h: P \rightarrow X$ be the relative Frobenius defined by $x \rightarrow u^{2}$. Then $h$ is a $G$-torsor. Thus by Construction 4.1, one has a functor

$$
h^{*}: \operatorname{Rep}_{k}(G) \rightarrow \operatorname{Strat}(X,-1) .
$$

We now compute that $h^{*}(k[G])$ is nothing but the -1 -stratified bundle defined in Example [3.3. Here $k[G]=k[v] /\left(v^{2}\right)$ is the regular representation of $G$. As in Example (3.3), let $X^{(i)}=k\left[x_{i}\right]$. Let $h^{*}(k[G])=\left(E^{(i)}, \sigma^{(i)}, i \in \mathbb{N}\right)$. As all schemes are affine, we confuse coherent sheaves with corresponding modules. Since $G_{\text {ét }}$ is trivial, by definition of $h^{*}$ we see that

$$
E^{(i)}=k\left[x_{i}\right] \otimes_{k} k[v] /\left(v^{2}\right) \quad \forall i \geq 1
$$

with

$$
\sigma^{(i)}: E^{(i)} \rightarrow F^{(i) *} E^{(i+1)}, \quad i \geq 1,
$$

induced by the identity map on $k[v] /\left(v^{2}\right)$. Then $E^{(0)}$ is by definition the $k[x]$ module of invariants of $k[u] \otimes_{k} k[v] /\left(v^{2}\right)$, where the action of $G=\operatorname{Spec} k[t] /\left(t^{2}\right)$ is defined by

$$
u \rightarrow u+t, \quad v \rightarrow v+t .
$$

Since $(u+v)^{2}=u^{2}=x$, one has $E^{(0)}=k[x] \cdot 1 \oplus k[x] \cdot(u+v)$. On $P$ we have an identification

$$
h^{*} E^{(0)}=k[u] \otimes_{k} k[v] /\left(v^{2}\right)
$$


defined by $\tau: 1 \mapsto 1 \otimes 1, u+v \mapsto u \otimes 1+1 \otimes v$. The map $\sigma^{(0)}$ is nothing but the pull back of $\tau$ via the isomorphism $X^{(-1)} \rightarrow P$ defined by

$$
k[u] \rightarrow k\left[x_{-1}\right], \quad u \rightarrow x_{-1} .
$$

We thus see that

$$
\sigma^{(0)}: k\left[x_{-1}\right] \cdot 1 \oplus k\left[x_{1}\right] \cdot(u+v) \longrightarrow k\left[x_{-1}\right] \otimes k[v] /\left(v^{2}\right)
$$

is defined by $1 \mapsto 1 \otimes,(u+v) \mapsto u \otimes 1+1 \otimes v$. It is then an elementary exercise to see that the stratified bundle $h^{*}(k[G])$ is isomorphic to the -1 -stratified bundle defined in Example 3.3 .

Lemma 4.4. The functor $h^{*}$ defined in (4.11) is k-linear, exact, compatible with the tensor structure and faithful.

Proof. As already recalled in the Properties 2.3 1), faithfulness follows from the remaining properties. On the other hand, $k$-linearity and compatibility with the tensor structures are straightforward. Exactness is proven by using Ver as in Theorem 3.43$)$. Indeed, Ver $\circ h^{*}$ with values in $\operatorname{Strat}\left(X^{(1)}\right)$ is obviously exact, while a sequence in $\operatorname{Strat}(X, \infty)$ is exact if and only if it remains exact after applying Ver.

If $\left(h_{i}: Y_{i} \rightarrow X, G_{i}, y_{i}\right)$ are objects in $\mathrm{N}(X, x)$ for $i=1,2$ and $\left(\psi: Y_{1} \rightarrow Y_{2}, \phi:\right.$ $\left.G_{1} \rightarrow G_{2}, y_{1} \rightarrow y_{2}\right)$ is a morphism in $\mathrm{N}(X, x)$, then Property 2.3 3) implies that $h_{2}^{*}=h_{1}^{*} \circ \phi^{*}$. On the other hand, the projective system of $\phi$ in $\mathrm{N}(X, x)$ induces an inductive system $\lim _{\mathrm{N}}(X, x), \phi^{*} \operatorname{Rep}_{k}(G)$ which is a Tannaka category, with the forgetful functor $F_{G}$ as the fiber functor. The Tannaka $k$-group-scheme $\operatorname{Aut}^{\otimes}\left(F_{G}\right)$ is simply $\lim _{\mathrm{N}(X, x), \phi} G$, which is precisely Nori's fundamental group-scheme $\pi^{N}(X, x)$. As in addition the construction is obviously functorial in $h$, we conclude:

Theorem 4.5. Let the notation be as in Construction 4.1. The functor $h^{*}$ defined in (4.11) for one object $(h: Y \rightarrow X, G, y)$ of $\mathrm{N}(X, x)$ induces a functor of Tannakian categories

$$
\mathfrak{h}^{*}:\left(\underset{\mathrm{N}(X, x), \phi^{*}}{\lim _{X}} \operatorname{Rep}_{k}(G), F_{G}\right) \rightarrow\left(\operatorname{Strat}(X, \infty), \omega_{x}\right)
$$

and the Tannaka-dual homomorphism of $k$-group-schemes

$$
\mathfrak{h}^{* \vee}: \pi^{\operatorname{alg}, \infty}(X, x) \rightarrow \pi^{N}(X, x),
$$

which is functorial in $X$.

The aim of the rest of the section is to show that the homomorphim $\mathfrak{h}^{* \vee}$ is faithfully flat and induces the profinite quotient homomorphism.

Proposition 4.6. Let $(Y \stackrel{h}{\rightarrow} X, G, y)$ be an object of $\mathrm{N}(X, x)$. The following conditions are equivalent:

1) The induced map $\pi^{\mathrm{alg}, \infty}(X, x) \rightarrow G$ (see (4.11) ) is an epimorphism.

2) The induced map $\pi^{N}(X, x) \rightarrow G$ is an epimorphism.

3) The functor $h^{*}$ in (4.11) is fully faithful, and its image is closed under taking subquotients in $\operatorname{Strat}(X, \infty)$. 
Proof. The equivalence (1) $\Leftrightarrow$ (3) follows from [2, Proposition 2.21]. Moreover, since by construction the map $\pi^{\text {alg, } \infty}(X, x) \rightarrow G$ factors through $\pi^{N}(X, x),(1) \Rightarrow$ (2) is obvious.

We show $(2) \Rightarrow(3)$. Let $\mathcal{C}$ denote the full subcategory of $\operatorname{Strat}(X, \infty)$ generated by subquotients in $\operatorname{Strat}(X, \infty)$ of objects which are in the image of $h^{*}: \operatorname{Rep}_{k}(G) \rightarrow$ $\operatorname{Strat}(X, \infty)$. Property 3$)$ is equivalent to saying that $h^{*}: \operatorname{Rep}_{k}(G) \rightarrow \mathcal{C}$ is an equivalenece of categories. By standard Tannaka formalism, $\mathcal{C}$ itself is a $k$-linear, abelian, rigid tensor subcategory of $\operatorname{Strat}(X, \infty)$. Thus $\left(\mathcal{C}, \rho_{x}\right)$ is a Tannaka subcategory of $\left(\operatorname{Strat}(X, \infty), \omega_{x}\right)$, where $\rho_{x}=\left.\omega_{x}\right|_{\mathcal{C}}$.

We now show that $h^{*}: \operatorname{Rep}_{k}(G) \rightarrow \mathcal{C}$ is an equivalence of categories. Let $H=\operatorname{Aut}\left(\rho_{x}\right)$ be the Tannaka $k$-group-scheme of $\left(\mathcal{C}, \rho_{x}\right)$. We claim that the induced homomorphism $H \rightarrow G$ is a closed immersion. This is equivalent (2, Proposition 2.21]) to saying that every object of $\mathcal{C}$ is a subquotient in $\mathcal{C}$ of an object in $h^{*}\left(\operatorname{Rep}_{k}(G)\right)$, which is true since by definition of $\mathcal{C}$ a subquotient in $\mathcal{C}$ of objects in $h^{*}\left(\operatorname{Rep}_{k}(G)\right)$ is the same as a subquotient in $\operatorname{Strat}(X, \infty)$ of objects in $h^{*}\left(\operatorname{Rep}_{k}(G)\right)$. We conclude in particular that $H$ is a finite group-scheme.

The fiber functor (in the sense of Deligne [1, 1.9]; see Properties [2.3 1)) $\omega_{X}$ : $\operatorname{Strat}(X, \infty) \rightarrow \operatorname{Coh}(X)$ defined by $\left(E_{i}, \sigma_{i}, i \in \mathbb{N}\right) \mapsto E_{0}$ restricts to the fiber functor $\rho_{X}: \mathcal{C} \rightarrow \operatorname{Coh}(X)$. One has a commutative diagram of functors

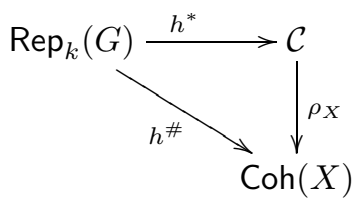

and, upon applying $i_{x}$, (4.1) implies that $i_{x} \circ h^{\#}=F_{G}$. By applying Theorem 2.4 we obtain a morphism

$$
\left(h_{H}: Y_{H} \rightarrow X, H, y_{H}\right) \rightarrow(h: Y \rightarrow X, G, y)
$$

in $\mathrm{N}(X, x)$. This in turn induces a factorization of $\pi^{N}(X, x) \rightarrow G$ as

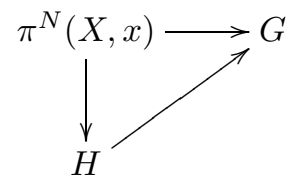

But $\pi^{N}(X, x) \rightarrow G$ is assumed to be an epimorphism. Thus $H \rightarrow G$ must be an epimorphism. Since it is also a closed immersion, we conclude that

$$
H \stackrel{\cong}{\rightarrow} G \text {. }
$$

In other words,

$$
h^{*}: \operatorname{Rep}_{k}(G) \stackrel{\cong}{\longrightarrow} \text {. }
$$

This finishes the proof.

Recall that $k$ is perfect.

Lemma 4.7. Let $G$ be a finite $k$-group-scheme, and let $h: Y \rightarrow X$ be a $G$-torsor. Then the following conditions are equivalent:

(i) $h$ admits a reduction (necessarily unique) of structure group to $G_{\text {red }}=$ $G_{\text {ét }} \subset G$. 
(ii) For every natural number $n$, there is a G-torsor $h_{n}: Y_{n} \rightarrow X^{(n)}$ which pulls back via $X \stackrel{F^{(0, n)}}{\longrightarrow} X^{(n)}$ to $h$.

Proof. We show (i) $\Rightarrow$ (ii). Let $h_{\text {ét }}: Y_{\text {ét }} \rightarrow X$ be a $G_{\text {ét-torsor which is a reduction }}$ of structure of $h$ for the closed embedding $G_{\text {ét }} \subset G$. Thus $Y=Y_{\text {ét }} \times_{G_{\text {ét }}} G$. The isomomorphism (4.3) induces a cartesian diagram

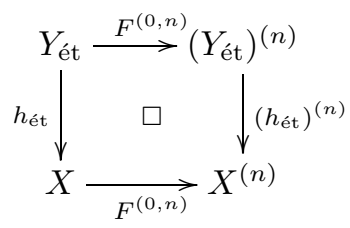

We set $Y_{n}=\left(Y_{\text {ét }}\right)^{(n)} \times_{G_{\text {ét }}} G$ and $h_{n}=\left(h_{\text {ét }}\right)^{(n)} \times_{G_{\text {ét }}} G$.

We show (ii) $\Rightarrow$ (i). For a large enough positive integer $n$, we consider the commutative diagram similar to (4.8):

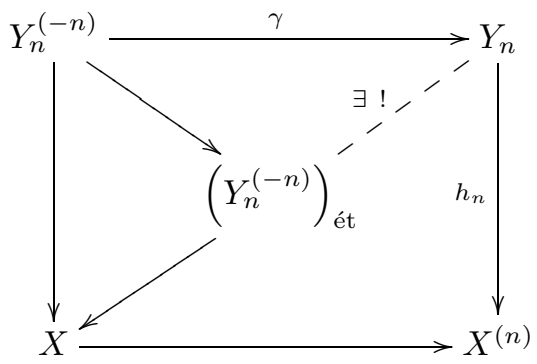

We explain the terms in the diagram: with Notation 3.1 one has $Y_{n}^{(-n)}=Y_{n} \otimes_{F_{k}^{-n}} k$. Thus $h_{n}$ induces $h_{n} \otimes_{F_{k}^{-n}} k: Y_{n}^{(-n)} \rightarrow\left(X^{(n)}\right)^{(-n)}=X$, which is a principal $G^{(-n)}$ bundle. The top horizontal map $\gamma$ is equivariant with respect to $G^{(-n)} \stackrel{F^{(-n, 0)}}{\longrightarrow} G$. Since $n$ is large, the image of $G^{(-n)} \rightarrow G$ is precisely $G_{\text {ét }} \subset G$. Therefore, $\gamma$ factors uniquely through $\left(Y_{n}^{(-n)}\right)_{\text {ét }}$. Via the identification $G_{\text {ét }}^{(-n)} \stackrel{F^{(-n, 0)}}{\longrightarrow} G_{\text {ét }}$, the

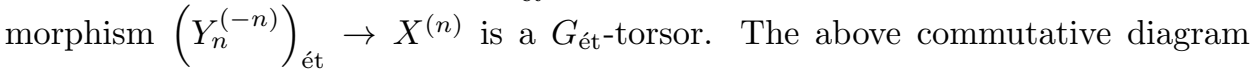
shows the existence of an equivariant map $\left(Y_{n}^{(-n)}\right)_{\text {ét }} \rightarrow Y_{n} \times_{X^{(n)}} X$. We conclude that the $G$-torsor $Y_{n} \times_{X^{(n)}} X \rightarrow X$ has a reduction of structure group to $G_{\text {ét }}$.

Theorem 4.8. Let the notation be as in Notation 3.1 and let $x \in X(k)$ be a rational point. Then the homomorphism $\mathfrak{h}^{* \vee}: \pi^{\text {alg, } \infty}(X, x) \rightarrow \pi^{N}(X, x)$ is the profinite quotient homomomorphism.

Proof. We have already shown in Proposition 4.6 that the homomorphism $\mathfrak{h}^{* \vee}$ is surjective. In order to show that $\mathfrak{h}^{* \vee}$ is the profinite completion homomorphism, we need to show that any epimorphism

$$
\phi: \pi^{\mathrm{alg}, \infty}(X, x) \rightarrow G,
$$

where $G$ is a $k$-finite group-scheme, factors through $\pi^{N}(X, x)$. This is equivalent to showing that given any finite Tannaka subcategory $\mathcal{T} \subset \operatorname{Strat}(X, \infty)$ (i.e. with $G=\operatorname{Aut}^{\otimes}\left(\mathcal{T}, \rho_{x}\right)$ finite $)$, where $\rho_{x}=\left.\omega_{x}\right|_{\mathcal{T}}$, there exists an object $(h: Y \rightarrow X, G, y)$ 
in $\mathrm{N}(X, x)$ such that $\mathcal{T}$ is the image of the functor $h^{*}$ constructed in (4.11). We do this in two steps.

Step 1. For each $n \geq 0$, we consider the fiber functor

$$
\omega_{X^{(n)}}: \operatorname{Strat}(X, \infty) \rightarrow \operatorname{Coh}\left(X^{(n)}\right), \quad\left(E^{(i)}, \sigma^{(i)}, i \in \mathbb{N}\right) \mapsto E^{(n)} .
$$

It restricts to a fiber functor

$$
P_{n}: \mathcal{T} \rightarrow \operatorname{Coh}\left(X^{(n)}\right) .
$$

Let $\delta: \operatorname{Rep}_{k}(G) \rightarrow \mathcal{T}$ be the equivalence of Tannaka categories defined by the inverse functor to the equivalence induced by $\rho_{x}$. Consider

$$
P_{n} \circ \delta: \operatorname{Rep}_{k}(G) \rightarrow \operatorname{Coh}\left(X^{(n)}\right) .
$$

By Theorem 2.4 we obtain $G$-torsors $\left(h_{n}: Y_{n} \rightarrow X^{(n)}\right)$ for every $n$ such that

$$
h_{n}^{\#}=P_{n} \circ \delta .
$$

Since the $G$-torsors thus obtained are unique up to isomorphism, the equality

$$
P_{n}=F^{(n) *} \circ P_{n+1}, \forall n \geq 1,
$$

implies that the torsor $h_{n+1}$ pulls back to $h_{n}$. Thus by Lemma 4.7, each $Y_{n}$ admits a reduction of structure group to $G_{\text {ét }} \subset G$ for all $n \geq 1$.

Step 2. Composing $\delta$ with the inclusion $\mathcal{T} \hookrightarrow \operatorname{Strat}(X, \infty)$ we obtain a functor from $\operatorname{Rep}_{k}(G) \rightarrow \operatorname{Strat}(X, \infty)$. We also have the functor $h_{0}^{*}: \operatorname{Rep}_{k}(G) \rightarrow \operatorname{Strat}(X, \infty)$ (see (4.11)) defined by the $G$-torsor $h_{0}: Y_{0} \rightarrow X$. In order to finish the proof we have to show that these two functors coincide. This is equivalent to saying that the following diagram of functors commutes:

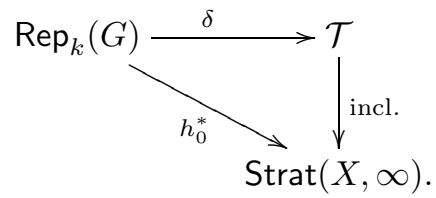

Let $V$ be an object of $\operatorname{Rep}_{k}(G)$. We will show that there is an isomorphism between $i(V)$ and $h_{0}^{*}(V)$, which is functorial in $V$. This will finish the proof. Let $\delta(V)=$ $\left(\delta(V)^{(n)}, \sigma^{(n)}, n \in \mathbb{N}\right)$ and $h_{0}^{*}(V)=\left(E^{(n)}, \tau^{(n)}, n \in \mathbb{N}\right)$.

We let $h_{n \text {,ét }}: Y_{n \text {,ét }} \rightarrow X^{(n)}$ be the $G_{\text {ét }}$-torsor induced by $h_{n}$ for $n \geq 1$. Note that by Construction 4.1 of the functor $h_{0}^{*}$, one has

$$
E^{(n)}=h_{n, \text { ét }}^{\#}(V) \forall n \geq 1 \text { and } E^{(0)}=h_{0}^{\#}(V) .
$$

On the other hand, by definition of the functors $P_{n}$,

$$
P_{n}(i(V))=i(V)^{(n)} .
$$

Thus by (4.21), one has

$$
i(V)^{(n)}=h_{n}^{\#}(V) \forall n \geq 0 .
$$

But as explained before, for every $n \geq 1, h_{n}: Y_{n} \rightarrow X^{(n)}$ admits a reduction of structure group to $G_{\text {ét }}$. Thus by Proposition 2.3 (3),

$$
h_{n}^{\#}(V)=h_{n \text {,ét }}^{\#}(V) \quad \forall n \geq 1 .
$$


Thus we conclude that

$$
i(V)=h_{0}^{*}(V) .
$$

If $\mathcal{T}$ is any $k$-linear, abelian, rigid tensor category, together with a neutral fiber functor $\omega: \mathcal{T} \rightarrow \operatorname{Vec}_{k}$, we denote by $\mathcal{T}^{\text {fin }}$ the full subcategory spanned by objects $E$ which have the property that the full tensor subcategory $\langle E\rangle \subset \mathcal{T}$ spanned by $E$ and its dual $E^{\vee}$ has a finite Tannaka group scheme $\operatorname{Aut}^{\otimes}\left(\langle E\rangle,\left.\omega\right|_{\langle E\rangle}\right)$. So by construction, Theorem 4.8 has the following consequence:

Corollary 4.9. With the notation as in Theorem 4.8, the full embedding

$$
\operatorname{Strat}(X, x)^{\mathrm{fin}} \subset \operatorname{Strat}(X, x)
$$

induces via the fiber functor $\omega_{x}$ the quotient homomorphism

$$
\pi^{\mathrm{alg}, \infty}(X,) \rightarrow \pi^{N}(X, x) .
$$

\section{ACKNOWLEDGMENTS}

This work started while the second author was a guest of the ERC Advanced Grant 226257 in Essen. Nguyên Duy Tân pointed out an inaccuracy in an earlier version of this work. The authors had useful discussions with Alexander Beilinson and Phùng Hô Hai, who came in February 2010 to the conference on Algebraic Geometry and Arithmetic in Essen, dedicated to the memory of Eckart Viehweg.

\section{REFERENCES}

[1] Deligne, P.: Catégories tannakiennes, The Grothendieck Festschrift, Vol. II, 111-195, Progr. Math., 87, Birkhäuser Boston, Boston, MA, 1990. MR1106898 (92d:14002)

[2] Deligne, P., Milne, J.: Tannakian Categories, Lecture Notes in Mathematics 900, 101-228, Springer-Verlag, 1982.

[3] dos Santos, J.-P.: Fundamental group schemes for stratified sheaves, J. Algebra 317 (2007), no. 2, 691-713. MR:2362937 (2008h:14045)

[4] dos Santos, J.-P.: Fundamental group-schemes in positive characteristic, Reports Oberwolfach, 2007.

[5] Esnault, H., Hai, P. H., Sun, X.: On Nori's fundamental group scheme. Geometry and dynamics of groups and spaces, 377-398, Progr. Math., 265, Birkhäuser, Basel, 2008. MR 2402410 (2009f:14091)

[6] Esnault, H., P. H. Hai: The fundamental groupoid scheme and applications, Annales de l'Institut Fourier 58 (2008), 2381-2412. MR2498355 (2010b:14046)

[7] Esnault, H., Mehta, V.: Simply connected projective manifolds in characteristic $p>0$ have no nontrivial stratified bundles, preprint 2009, 12 pages.

[8] van der Geer, G., Moonen, B.: Abelian varieties, http://staff.science.uva.nl/bmoonen/ boek/BookAV.html.

[9] Gieseker, D.: Flat vector bundles and the fundamental group in non-zero characteristics, Ann. Scu. Norm. Sup. Pisa, 4. série, tome 2, no. 1 (1975), 1-31. MR0382271 (52:3156)

[10] Nori, M.: The Fundamental Group-Scheme, Proc. Ind. Acad. Sc. Math. Sci. 91 no. 2 (1982), 73-122. MR682517 (85g:14019)

Department of Mathematics, Universität Duisburg-Essen, 45117 Essen, Germany

E-mail address: esnault@uni-due.de

School of Mathematics, Tata Institute of Fundamental Research, Homi Bhabha Road, Colaba, Mumbai 400005, India

E-mail address: amit@math.tifr.res.in 